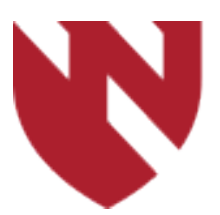

\title{
Does Immediate Postoperative Opioid Consumption Correlate with Long-term Outcomes in Patients Undergoing One and Two Level Instrumented Posterior Lumbar Fusions?
}

\author{
Emmett J. Gannon \\ University of Nebraska Medical Center \\ Evan P. Larson \\ Univeristy of Nebraska Medical Center \\ Zachary C. Bailey \\ University of Nebraska Medical Center \\ Sydney Powers \\ University of Nebraska Medical Center \\ Scott A. Vincent \\ University of Nebraska Medical Center
}

Tell us how you used this information in this short survey.

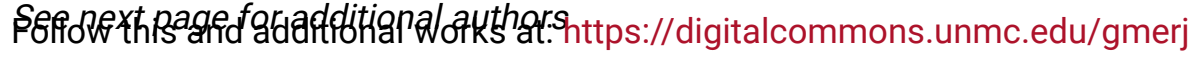

Part of the Higher Education Commons, and the Medicine and Health Sciences Commons

\section{Recommended Citation}

Gannon, E. J., Larson, E. P., Bailey, Z. C., Powers, S., Vincent, S. A., Cornett, C. A., , Lyden, L. Does Immediate Postoperative Opioid Consumption Correlate with Long-term Outcomes in Patients Undergoing One and Two Level Instrumented Posterior Lumbar Fusions?. Graduate Medical Education Research Journal. 2020 Sep 29; 2(1). https://digitalcommons.unmc.edu/gmerj/vol2/iss1/37 
Does Immediate Postoperative Opioid Consumption Correlate with Long-term Outcomes in Patients Undergoing One and Two Level Instrumented Posterior Lumbar Fusions?

Creative Commons License

(c) $(1) \Theta$

This work is licensed under a Creative Commons Attribution-Noncommercial-No Derivative Works 4.0 License.

\section{Authors}

Emmett J. Gannon, Evan P. Larson, Zachary C. Bailey, Sydney Powers, Scott A. Vincent, Chris A. Cornett, and Liz Lyden 
Conclusion: Perioperative TXA administration was associated with reduced mean operative time and decreased intraoperative estimated blood loss. While not statistically significant, these results may be clinically significant. Larger, higher level studies are required for further investigation.

https://doi.org/10.32873/unmc.dc.gmerj.2.1.035

\section{Does Immediate Postoperative Opioid Consumption Correlate With Long-Term Outcomes in Patients Undergoing One and Two Level Instrumented Posterior Lumbar Fusions?}

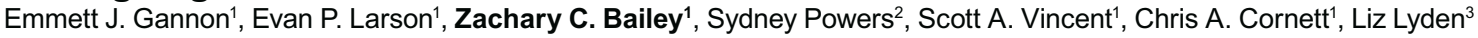

${ }^{1}$ University of Nebraska Medical Center, Department of Orthopaedic Surgery and Rehabilitation

${ }^{2}$ University of Nebraska Medical Center, College of Medicine

${ }^{3}$ University of Nebraska Medical Center, College of Public Health, Department of Biostatistics

Mentor: Chris Cornett

Program: Orthopaedic Surgery and Rehabilitation

Type: Original Research

Background: Opioid analgesics are routinely used following spine surgery. Preoperative and chronic postoperative use are associated with worse postoperative pain control and functional outcomes. Little is known about the effects of immediate postoperative use on long term outcomes. The purpose of this study is to investigate the potential relationship between increased opioid use immediately postoperatively and patient outcomes.
Methods: A retrospective review of patients undergoing instrumented 1 or 2-level posterior lumbar fusion surgery by a single surgeon was performed. Pearson correlation coefficients were used to evaluate associations between patient outcomes and immediate postoperative opioid use. Exclusion criteria included previous lumbar surgery, indications other than degenerative, and patients $<19$ years old. A total of 152 patients were included.

Results: Among 94 patients undergoing 1-level fusion, there was a significant inverse relationship between six month Short Form36 Bodily Pain and postoperative day (POD) 1,2 and total opioid use $(\mathrm{p}=0.0328, \mathrm{p}=0.0283$, and $\mathrm{p}=0.0205$ ). A significant inverse relationship was also found between 1 year Short Form-36 Physical Function and POD 1 and 2 opioid use $(\mathrm{p}=0.0415, \mathrm{p}=0.0200)$ and between 1 year Short Form-36 Bodily Pain and POD 2 opioid use ( $\mathrm{p}=0.0099)$. No correlation existed between amount of opioids consumed and outcomes after 2-level posterior lumbar fusion procedures.

Conclusion: The amount of opioid analgesics consumed in the immediate postoperative period may be helpful in predicting patient outcomes at 6 months and 1 year following 1-level posterior lumbar fusion. These findings, however, must be interpreted in context with the limitations inherent to a retrospective study.

https://doi.org/10.32873/unmc.dc.gmerj.2.1.036

\section{Rare Case of Traumatic Tricuspid Valve Injury in Patient with Sinus Venosus Atrial Septal Defect (ASD)}

Rishi Batra', Laura Newton ${ }^{1}$, Nicholas Markin², Samuel Cemaj', Zachary Bauman ${ }^{1}$

${ }^{1}$ University of Nebraska Medical Center, Department of Surgery

${ }^{2}$ University of Nebraska Medical Center, Department of Anesthesiology

Mentor: Zach Bauman

Program: General Surgery

Type: Case Report

Background: A 69-year-old male with history of congenital ASD repair as a child, atrial fibrillation and left ventricular dysfunction, presented as the restrained passenger of a motor vehicle crash. Initially complaining of left-sided chest pain, he was found to have left rib fractures 5-8 with flail physiology as well as a left tibial plateau and ulnar fracture. Due to flail chest physiology, open reduction and internal fixation (ORIF) of the ribs was scheduled.

Methods: After transthoracic echocardiogram (TTE) was completed, he was taken for ORIF. Upon intubation, he became acutely hypoxemic. Immediate left chest tube was placed, however, his clinical status remained unchanged. Emergent bronchoscopy was performed without resolution of the hypoxemia. Intraoperative transesophageal
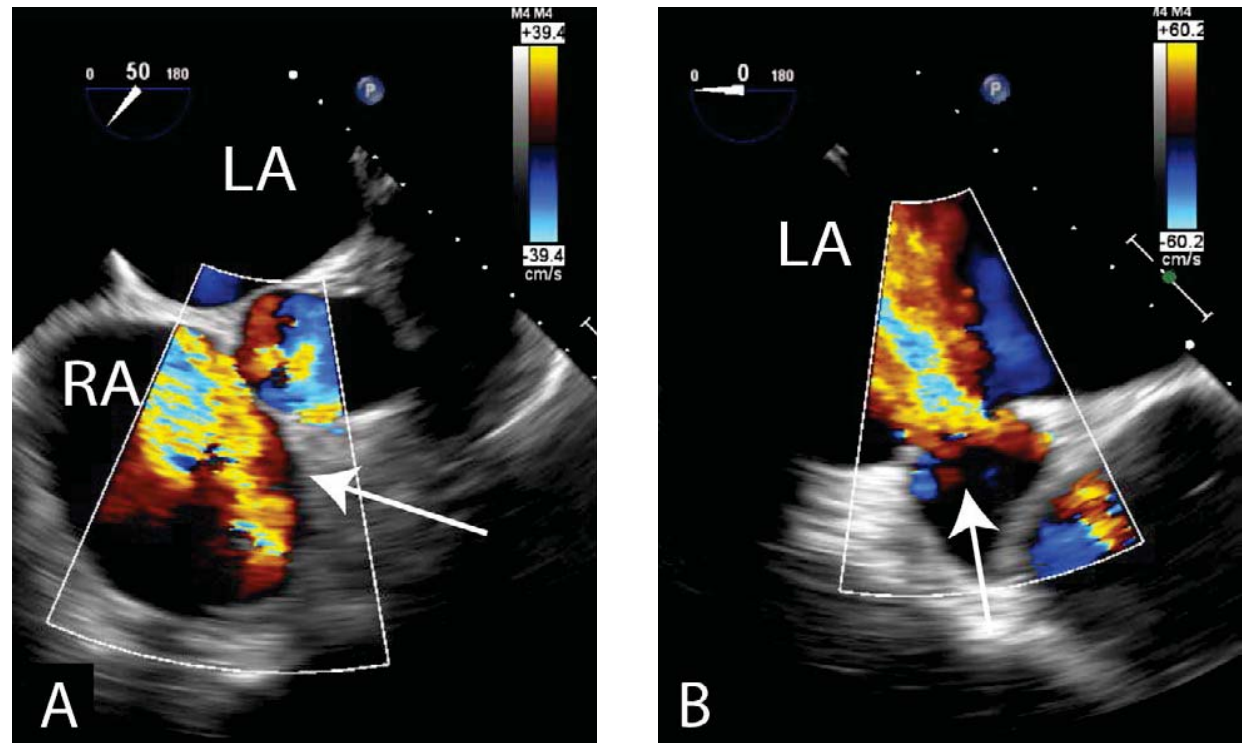

Figure 1A. Transesophageal echocardiogram showing the midesophageal modified right ventricular inflow view. There is severe tricuspid regurgitation (marked with arrow).

Figure 1B. Transesophageal echocardiogram showing a lower esophageal view of the inferior sinus venosus atrial septal defect. This image shows a high-velocity jet with right-to-left shunting. This is the result of the traumatic severe TR and the jet is directed at the sinus venosus atrial septal defect causing the right-to-left shunting (measuring $75 \mathrm{~mm} 2 \&<10 \mathrm{~mm} 2$ ). 